\title{
Surgical approach and orthodontic treatment of mandibular condylar osteochondroma
}

\author{
So Jin Yang ${ }^{\mathrm{a}, \mathrm{b}}$ (1) \\ Nam Hyung Chung ${ }^{\mathrm{a}, \mathrm{b}}$ \\ Jong Ghee Kim ${ }^{\mathrm{a}, \mathrm{b}, \mathrm{c}}$ \\ Young-Mi Jeon ${ }^{\mathrm{ab}, \mathrm{c}}$ (1)
}

${ }^{a}$ Department of Orthodontics, School of Dentistry, Jeonbuk National University, Jeonju, Korea

${ }^{b}$ Institute of Oral Biosciences, School of Dentistry, Jeonbuk National University, Jeonju, Korea

'Research Institute of Clinical Medicine of Jeonbuk National UniversityBiomedical Research Institute of Jeonbuk National University Hospital, Jeonju, Korea
Osteochondroma is a common benign tumor of bones, but it is rare in the mandibular condyle. With its outgrowth it manifests clinically as deviation of the mandible limitation of mouth opening, and facial asymmetry. After the tumor is diagnosed on the basis of clinical symptoms and radiographic examination including cone-beam computed tomography (CBCT) analysis, an appropriate surgery and treatment plan should be formulated. Herein, we present the case of a 44-year-old female patient who visited our dental hospital because her chin point had been deviating to the left side slowly but progressively over the last 3 years and she had difficulty masticating. Based on CBCT, she was diagnosed with skeletal Class 111 malocclusion accompanied by osteochondroma of the right mandibular condyle. Maxillary occlusal cant with the right side down was observed, but it was confirmed to be an extrusion of the molars associated with dental compensation. Therefore, after intrusion of the right molars with the use of temporary anchorage devices, sagittal split ramus osteotomy was used to remove the tumor and perform orthognathic surgery simultaneously. During 6 months after the surgery, continuous bone resorption and remodeling were observed in the condyle of the affected side, which led to a change in occlusion. During the postoperative orthodontic treatment, intrusive force and buccal torque were applied to the molars on the affected side, and a proper buccal overjet was created. After 18 months, СBCT revealed that the rate of bone absorption was continuously reduced, bone corticalization appeared, and good occlusion and a satisfying facial profile were achieved.

[Korean J Orthod 2020;50(3):206-215]

Key words: Osteochondroma, Condyle remodeling

Received December 10, 2018; Revised March 25, 2019; Accepted March 29, 2019.

Corresponding author: Young-Mi Jeon.

Professor, Department of Orthodontics, Jeonbuk National University Hospital, School of Dentistry, Jeonbuk National University, 20 Geonji-ro, Deokjin-gu, Jeonju 54907, Korea. Tel+82-63-250-2123 e-mail young@jbnu.ac.kr

How to cite this article: Yang SJ, Chung NH, Kim JG, Jeon YM. Surgical approach and orthodontic treatment of mandibular condylar osteochondroma. Korean J Orthod 2020; 50:206-215.

(C) 2020 The Korean Association of Orthodontists.

This is an Open Access article distributed under the terms of the Creative Commons Attribution Non-Commercial License (http://creativecommons.org/licenses/by-nc/4.0) which permits unrestricted non-commercial use, distribution, and reproduction in any medium, provided the original work is properly cited. 


\section{INTRODUCTION}

Osteochondroma is a common benign tumor in the ilium but it is rare in the craniofacial region. This is because most craniofacial bones originate from intramembranous ossification.' Osteochondroma in the craniofacial region incurs mainly in the mandibular coronoid process and rarely in the condylar process. ${ }^{2,3}$ Osteochondroma proliferation often results in a protrusion from the bone surface that is surrounded by cartilage. ${ }^{4}$

According to Henry et al. ${ }^{5}$ and Karras et al., ${ }^{6}$ osteochondroma in the ilium mainly occurs in teenagers and stops proliferating once their growth is complete, whereas osteochondroma in the mandibular condyle occurs in adults with a mean age of 39.6 years, and continues to proliferate. ${ }^{7}$ It has also been reported that osteochondroma in the ilium was more common in men, whereas osteochondroma in the mandibular condyle was more common in women. ${ }^{8}$ Osteochondroma requires differential diagnosis from osteoma, benign osteoblastoma, and chondroma. When it occurs in the facial bone region, it proliferates slowly, and almost never exhibits malignant conversion. ${ }^{9}$ When it occurs in the mandibular condyle, it causes mouth opening displacement and functional disorders, malocclusion, and severe facial asymmetry. ${ }^{10}$

In order to diagnose osteochondroma, clinical symptoms, radiological findings, and cone-beam computed tomography (CBCT) imaging should be comprehensively evaluated. Based on the results of this multi-faceted analysis, a strategic treatment plan should be established. Osteochondroma in the condylar process is usually treated via condylectomy as an extraoral approach. In the present case, the tumor was removed while bilateral sagittal split ramus osteotomy (BSSRO) was performed to improve mandibular prognathism, as an intraoral approach. By applying mandibular setback via BSSRO and simultaneously removing the tumor, it was possible to achieve proper occlusion. In addition, with comprehensive orthognathic-orthodontic treatment, the skeletal discrepancy was corrected, and this led to improvements in facial symmetry and aesthetic appearance.

\section{DIAGNOSIS AND ETIOLOGY}

A 44-year-old female patient visited our dental hospital due to mandibular deviation to the left that had become distinguishable approximately 3 years prior and was causing her difficulty with mastication. She had no remarkable relevant medical history prior to that, but she had undergone treatment with a splint at a different dental clinic. Mandibular left displacement, anterior crossbite, and buccal crossbite of the left molars were observed (Figure 1). However, her facial midline and maxillary dental midline were almost consistent. Aside from that, she had root rest of the upper first premolar, generalized gingival recession, and mandibular anterior
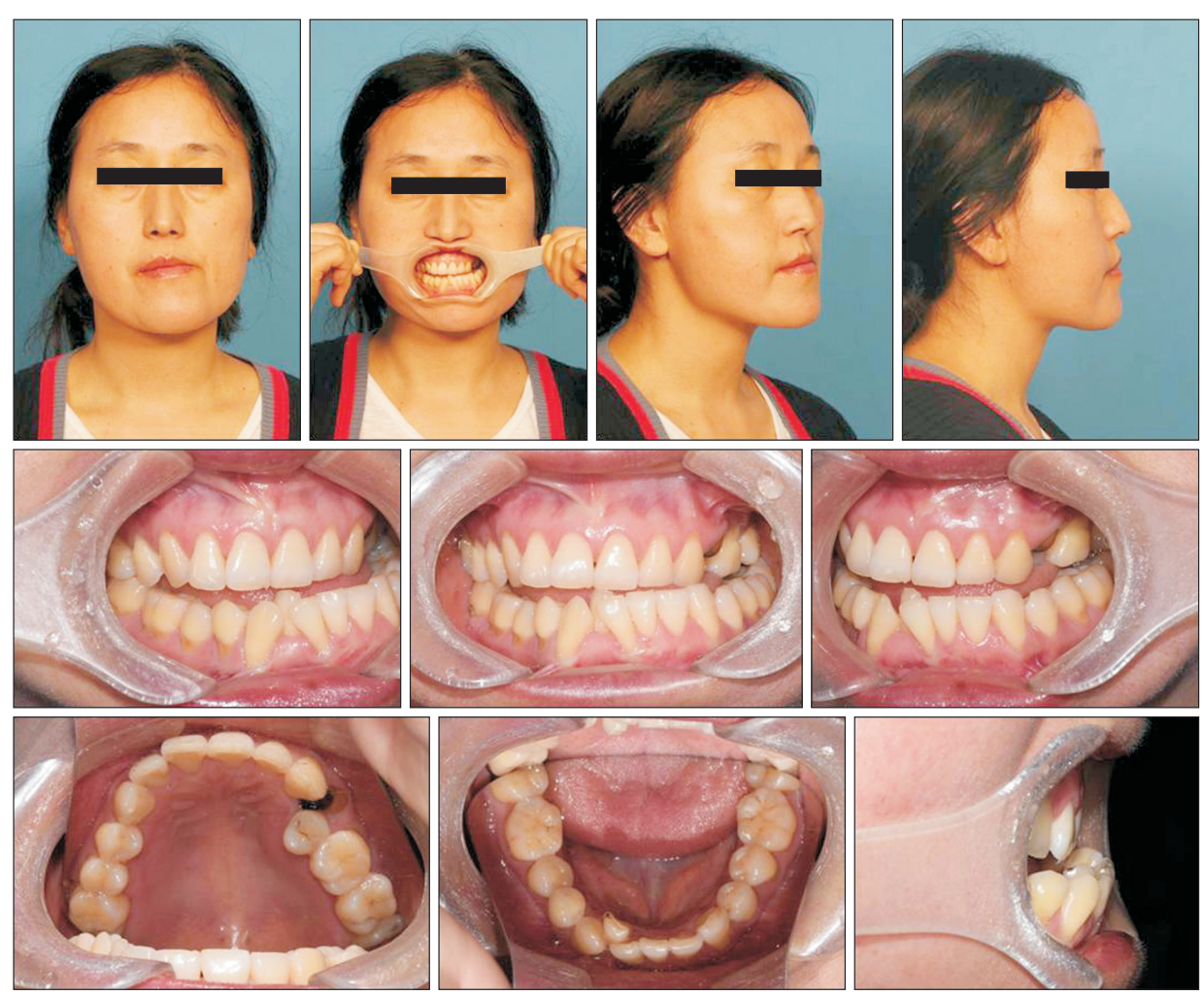

Figure 1. Pretreatment facial and intraoral photographs. 
crowding. Mandibular prognathism caused a concave profile, and her upper and lower lips were recessed back from E-line. In her initial study model, a Class 111 molar relationship and an anterior crossbite were apparent (Figure 2). In a temporal mandibular joint examination, she reported no pain in either condyle, but exhibited a clicking sound in her right temporal mandibular joint.

On panoramic view, a radiopaque mass with a relatively well-defined border was evident in the right condyle (Figure 3), and CBCT revealed a bone tumor that had proliferated in the antero-mesial region of the right condyle. The inner side of the mass exhibited an

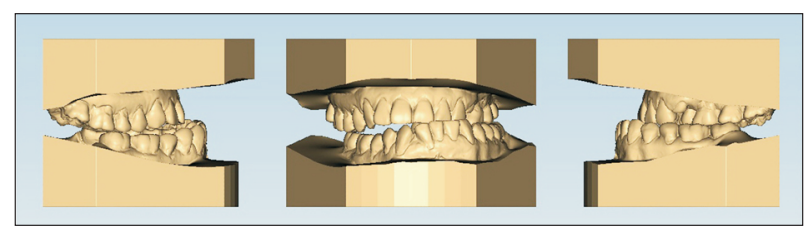

Figure 2. Pretreatment dental models. irregular bony trabecula, and the osteochondroma was continuous with the underlying apparent cortical bone (Figure 4A). The associated articular eminence and glenoid fossa were deformed in a manner corresponding with the shape of the lesion. In addition, the left mandibular condyle exhibited erosion in its upper front side,

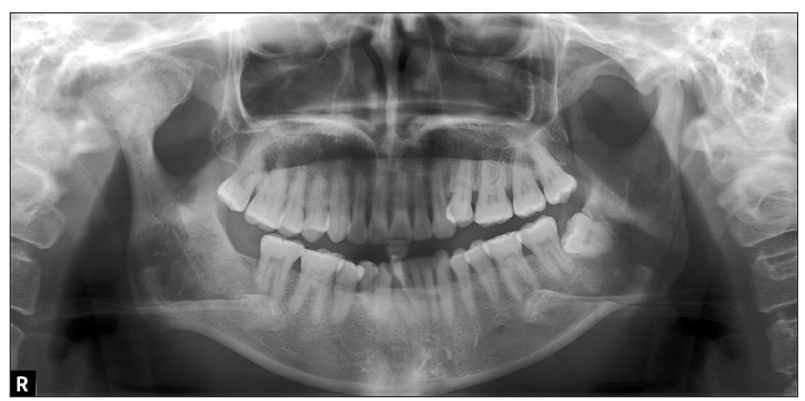

Figure 3. A pretreatment panoramic radiograph. The panoramic view depicted an irregular bony mass on the mandibular right condyle.
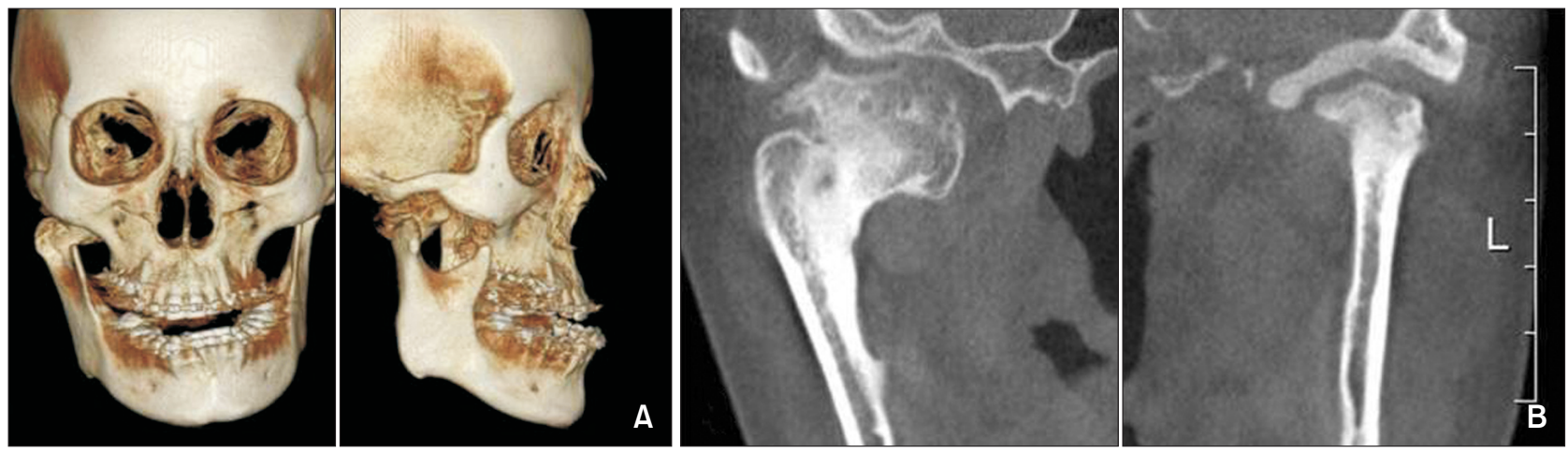

Figure 4. Preoperative cone-beam computed tomography (CBCT) images. A, Frontal and lateral view three-dimensional images. B, CBCT images focused on the condylar process. Coronal view showing the mass extending medially into the infratemporal fossa.
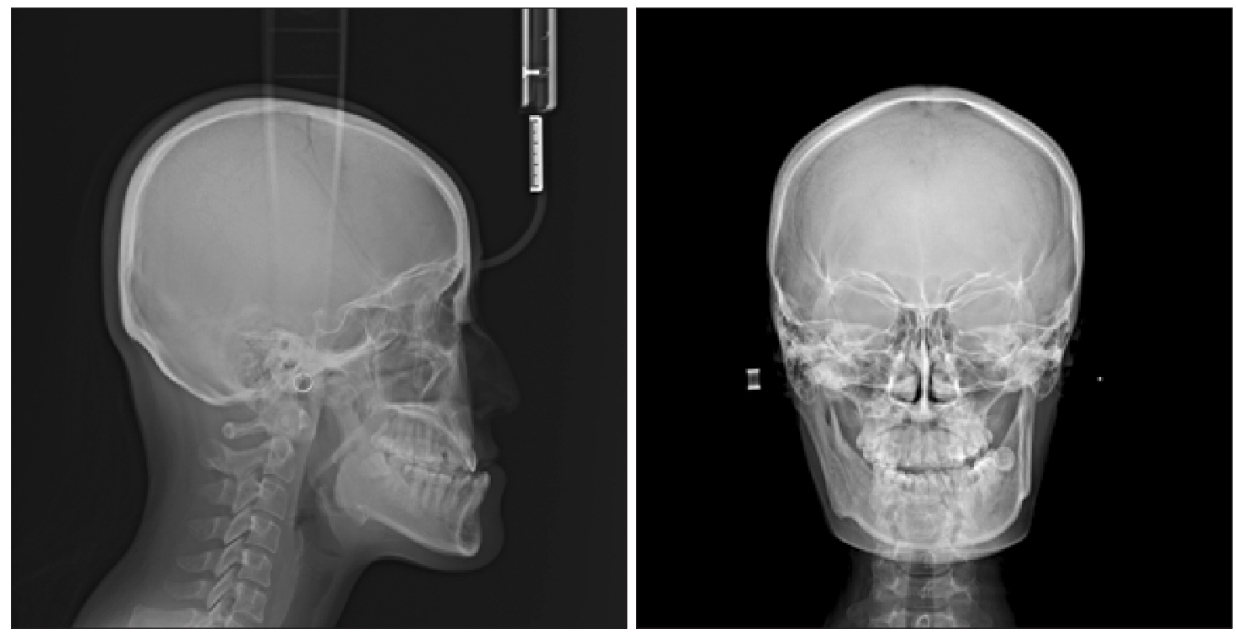

Figure 5. Pretreatment cephalograms. 
and osteoarthrosis was apparent (Figure 4B).

On lateral cephalogram, the ANB angle was $-4.9^{\circ}$ and Wits appraisal value was -16.9 , thus she exhibited skeletal Class 111 (Figure 5). The interincisal angle (IIA) was $141.9^{\circ}$, which is above the normal range (Table 1). On frontal cephalogram, the menton from the midsagittal plane was displaced approximately $10^{\circ}$ to the left and vertical difference of right and left antegonion was prominent, but there was no significant difference in the jugular points. In addition, CBCT suggested that canting of the maxillary occlusal plane was a result of dental compensation of the extruded maxillary right molars, not skeletal factors. Accordingly, the patient was diagnosed with a skeletal Class 111 jaw-base relationship with facial asymmetry caused by right condylar osteochondroma.

\section{TREATMENT OBJECTIVES}

The treatment objectives were to (1) improve facial symmetry by removing the osteochondroma; (2) correct the anterior crossbite and achieve proper overjet and overbite; and (3) achieve acceptable masticatory function and good occlusion.

\section{TREATMENT ALTERNATIVES}

In the present patient, a buccal crossbite on the left side and maxillary right molar extrusion were reported in the initial record. However, in frontal cephalography and $\mathrm{CBCT}$, there was almost no vertical difference between the jugular points in the maxilla, and the width of the first molar was almost within the normal range. Therefore, vertical and transverse discrepancies were considered to have resulted from dental compensation due to the osteochondroma in the right condyle. In the
Table 1. Cephalometric summary

\begin{tabular}{lcc}
\hline \multicolumn{1}{c}{ Measurement } & Pre-treatment & Post-treatment \\
\hline SNA $\left({ }^{\circ}\right)$ & 79.8 & 78.5 \\
SNB $\left({ }^{\circ}\right)$ & 84.7 & 75.6 \\
ANB $\left({ }^{\circ}\right)$ & -4.9 & 2.7 \\
Wits $(\mathrm{mm})$ & -16.9 & -1.8 \\
APDI $\left(^{\circ}\right)$ & 101.2 & 84.1 \\
Sum $\left({ }^{\circ}\right)$ & 389.6 & 397.5 \\
SN-GoGn $\left({ }^{\circ}\right)$ & 29.5 & 38.5 \\
FMA $\left(^{\circ}\right)$ & 24.0 & 32.9 \\
OP-GoMe $\left(^{\circ}\right)$ & 10.7 & 19.0 \\
ODI $\left({ }^{\circ}\right)$ & 64.8 & 74.5 \\
U1 to SN $\left({ }^{\circ}\right)$ & 96.1 & 97.5 \\
IMPA $\left({ }^{\circ}\right)$ & 92.4 & 93.2 \\
Interincisal angle $\left({ }^{\circ}\right)$ & 141.9 & 129.9 \\
Upper lip to E-plane $(\mathrm{mm})$ & -6.2 & -1.6 \\
Lower lip to E-plane $(\mathrm{mm})$ & -2.2 & -2.9 \\
\hline
\end{tabular}

SNA, Sella-nasion-A point; SNB, sella-nasion-B point; ANB, sagittal jaw relationship; Wits, "Wits value" —an indicator of anteroposterior disharmony between the maxilla and the mandible; APDI, anteroposterior dysplasia indicator; Sum, sum of saddle angle, articular angel, and gonial angle; SN-GoGn, sella-nasion to mandibular plane angle; FMA, Frankfort mandibular plane angle; OP-GoMe, angle between the occlusal plane and the mandibular plane; ODI, overbite depth indicator; U1 to SN, angle between the maxillary central incisor and the sella-nasion; IMPA, lower incisor mandibular plane angle; Upper lip to E-plane, distance from the upper lip to the E line; Lower lip to E-plane, distance from the lower lip to the $\mathrm{E}$ line.
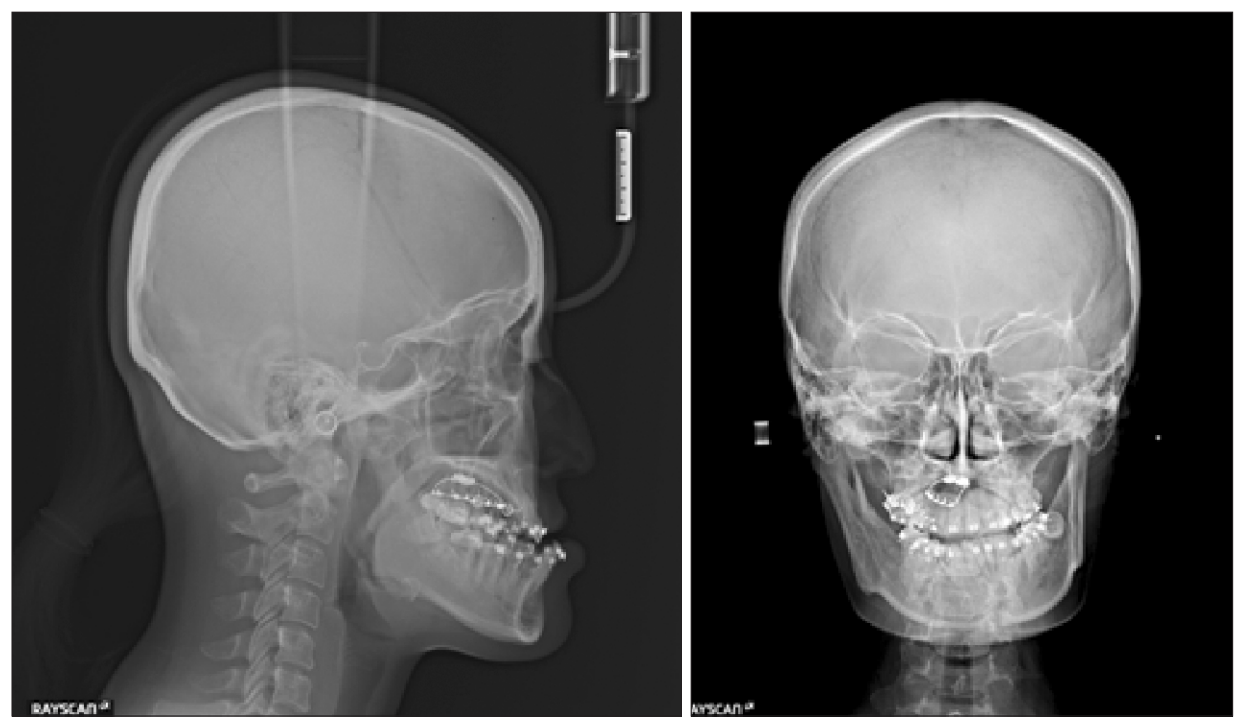

Figure 6. Cephalograms before surgery. 
current case, we decided not to include the maxilla in the operation, but to perform mandibular surgery with concurrent removal of the osteochondroma.

Osteochondroma of the mandibular condyle has traditionally been removed via total condylectomy in order to prevent recurrence, but this may result in the loss of vertical dimension, and occlusal interference. "Recently, patients treated for osteochondroma have experienced good outcomes even after removal of the lesion via conservative techniques. As shown in the present case, if osteochondroma proliferation is restricted to the surface of the condylar process, it is possible to utilize conservative surgical excision of the lesion in conjunction with orthognathic surgery, depending on the purpose of the skeletal improvement.

In terms of surgical approach according to the area of condyle osteochondroma, a preauricular approach is most commonly used, and either a submandibular or
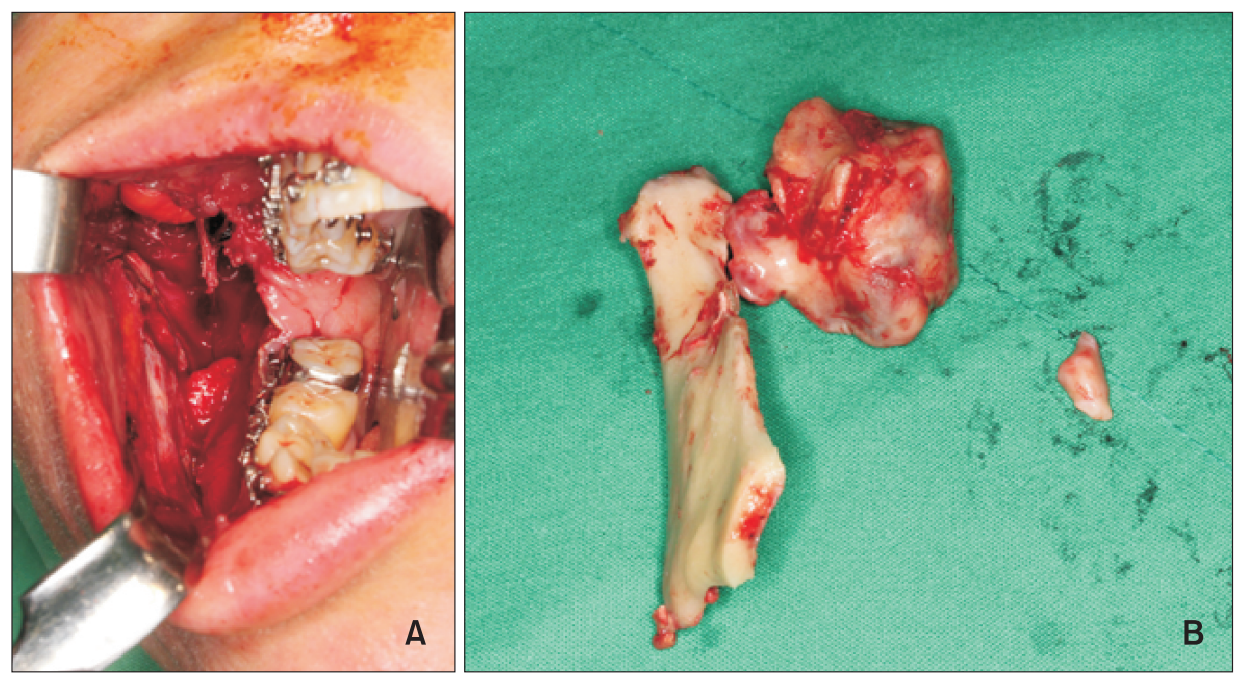

Figure 7. Intraoperative clinical photographs. A, Surgical procedure via an intraoral approach. $\mathbf{B}$, The osteochondroma was removed with preservation of the condyle.

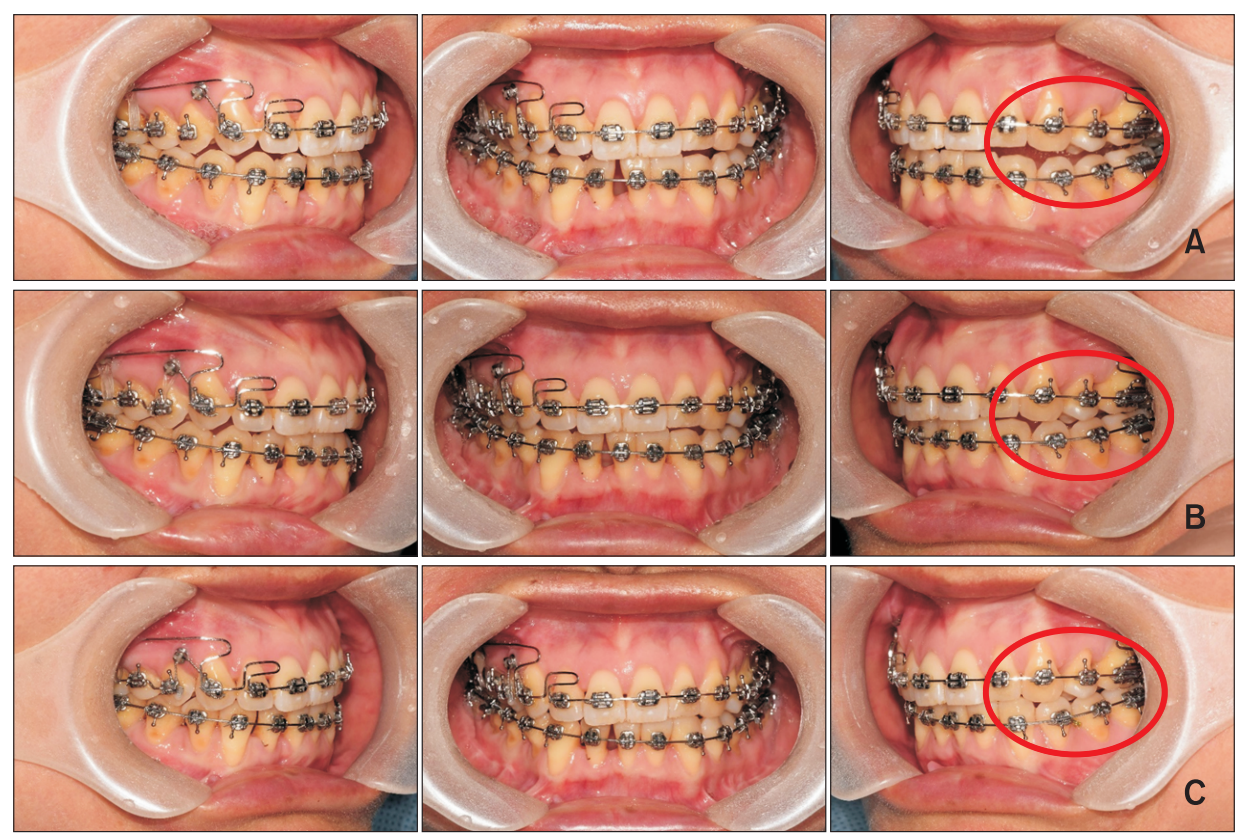

Figure 8. Progress of orthodontic treatment. A, Intraoral photographs 6 months after the surgery. Premature contact of the right molars and open bite of the left molars (circle) occurred. B, Intraoral photographs 10 months after the surgery. The open bite of the left molars (circle) had gradually reduced in conjunction with the use of temporary anchorage de-

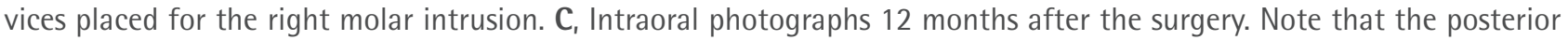
open bite on the left side (circle) was improved and a proper buccal overjet was achieved. 
a retromandibular approach is also used. ${ }^{5}$ If the tumor is large, a temporalis flap can be formed or additional zygomatic arch excision surgery can be performed to ensure visibility, or an intraoral approach can be used. ${ }^{7}$ When condylar osteochondroma is removed via a preauricular approach, there is a risk of non-aesthetic facial scarring and facial nerve (temporal and zygomatic branches) damage. The current patient did not want a scar on her face, and the lesion was more than $30 \mathrm{~mm}$ in diameter. For these reasons, to resolve the mandibular prognathism, BSSRO was performed via an intraoral approach to take the right proximal segment out of the mouth in order to remove the tumor, then it was repositioned after the condyle was reshaped.

\section{TREATMENT PROGRESS}

To correct the extrusion of right molars by dental compensation before surgery, along with leveling, intrusion in palatal and buccal sides was applied with temporary anchorage devices (TADs). A minimum of 5 months of pre-surgical orthodontic treatment was undertaken and a surgical plan was established after measuring the size of the tumor accurately via СВCT. Just before the surgery, both cephalogram analysis and model surgery were conducted to make a final wafer (Figure 6).

To achieve an acceptable occlusion with a skeletal
Class 1 relationship, BSSRO was performed and the right proximal segment was taken out of the mouth to remove the osteochondroma in conjunction with condylar reshaping (Figure 7). The removed mass had approximate dimensions of $33 \times 31 \times 28 \mathrm{~mm}$. The mandible was moved backward $17 \mathrm{~mm}$ on the right side and 8.5 $\mathrm{mm}$ on the left side. After the surgery, the wafer was placed stably. The facial swelling was more prominent on the right side than on the left side. Intermaxillary fixation was conducted for 3 weeks, and mouth opening training was initiated thereafter.

During the postoperative orthodontic treatment, the mandibular right incisor exhibited poor periodontal condition so it was extracted. During the observation period after the surgery, the patient underwent continuous bone resorption and remodeling of the condyle for 6 months. Right posterior premature contact and shallow

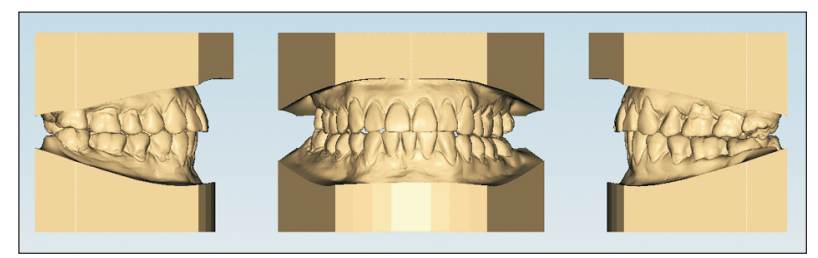

Figure 10. Post-treatment dental models.
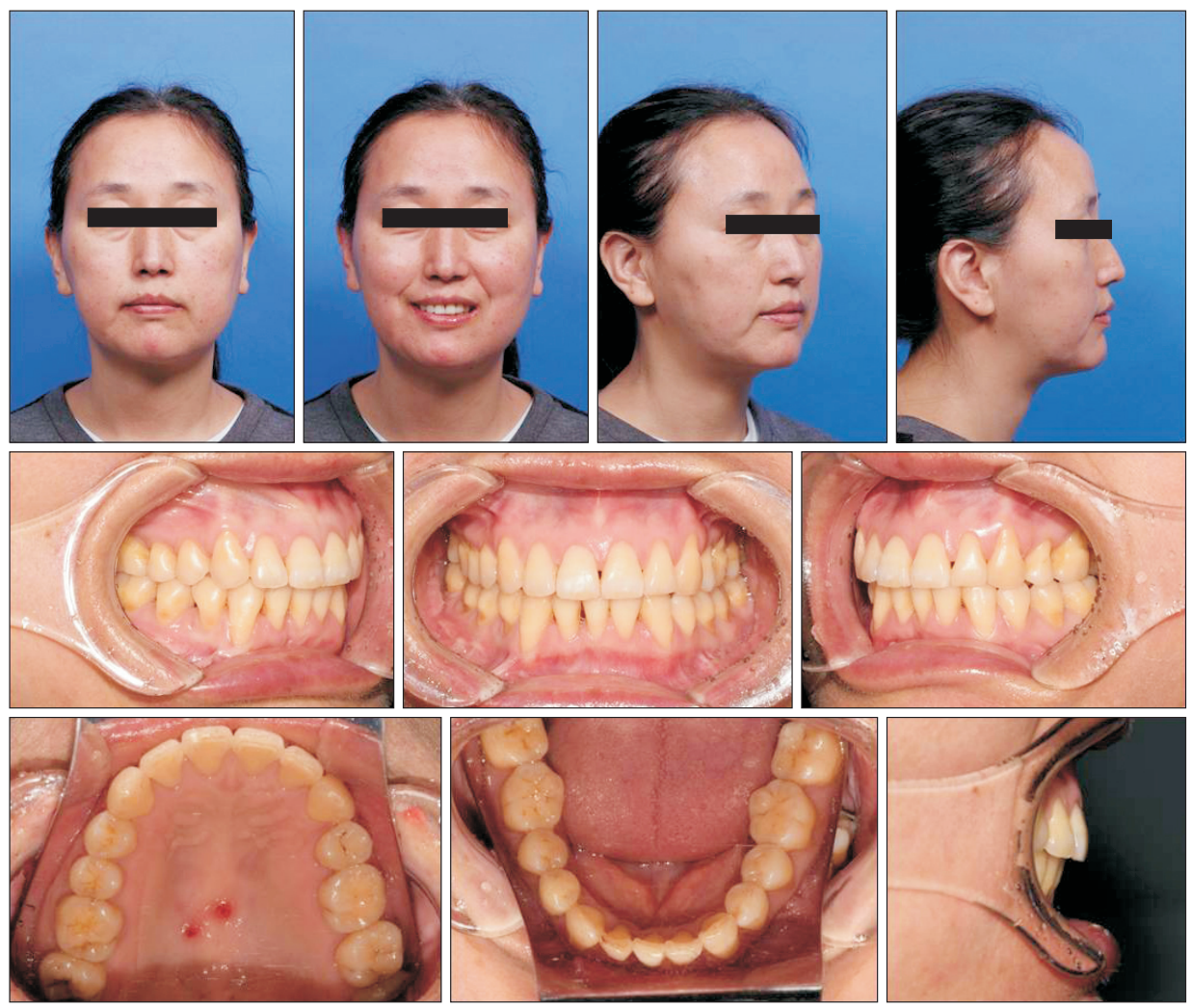

Figure 9. Post-treatment facial and intraoral photographs. 
buccal overjet were observed, as were left posterior bite opening and large buccal overjet, and the anterior overjet gradually increased (Figure 8).

To manage the change in occlusion resulting from condylar remodeling, wire bending was applied for distalization and intrusion of the maxillary right second molar, segmented stainless steel wire was inserted from the maxillary right first premolar to the first molar, and intrusive force and buccal torque were applied using TADs. For the left molars, elastic-based extrusion mechanics were applied during the postoperative orthodontic treatment period. The premature contact of the right molars and the open bite of the left molars that were evident 6 months postoperatively had evidently reduced somewhat by 10 months postoperatively, and 1 year after the operation the patient exhibited a proper buccal overjet and good occlusion (Figure 8). Eighteen months later, radiographic examination revealed slowing of bone resorption of the condyle, and cortical bone formation was evident. Because stable occlusion had been achieved clinically, the orthodontic appliance was removed.

\section{TREATMENT RESULTS}

At the conclusion of active orthodontic treatment, in terms of the molar relationship the right side exhibited Class 1 and the left side exhibited Class 11, there was an appropriate overjet and overbite, and functional occlusion was obtained (Figures 9 and 10). Facial asymmetry and facial profile were dramatically improved. In lateral cephalometric analysis after the treatment, most values were almost within normal ranges (Table 1). A skeletal Class 1 jaw relationship was achieved (ANB 2.7 ) and an adequate IIA $129.9^{\circ}$ was established. The position of the condyle was repositioned upward and backward after removal of the tumor, as determined by superimposing the pre- and post-orthodontic treatment lateral cephalograms (Figure 11). On frontal cephalogram, the mandibular deviation to the left side was also remarkably improved, and acceptable occlusion was evident (Figure 12). The patient had no masticatory disturbance and the bilateral mouth corners were symmetrical while smiling (Figure 9).

Miyamoto et al. ${ }^{12}$ reported that within approximately 3 months after condylectomy, condyle remodeling and joint cartilage were created. Change in the right condyle of the present patient was observed straight after the

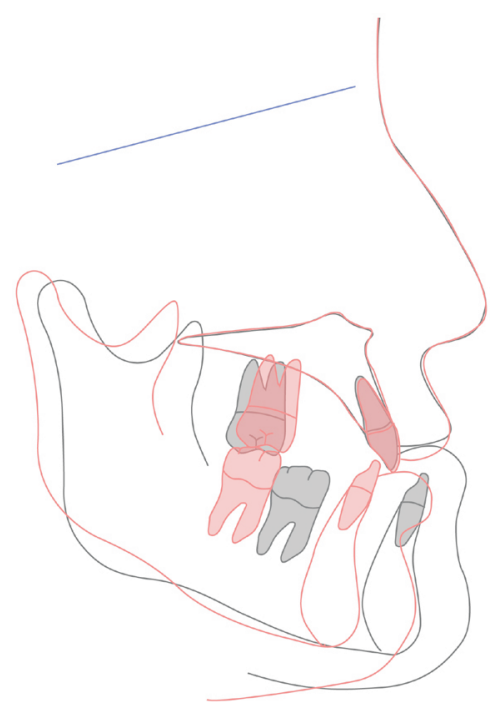

Figure 11. Initial (black line) and final (red line) cephalometric tracings superimposed on the sella-nasion plane (blue line).

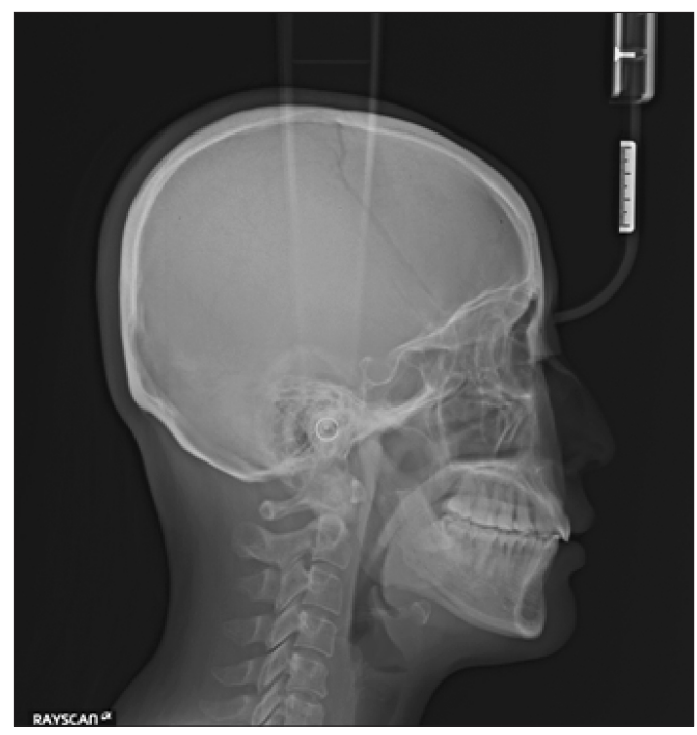

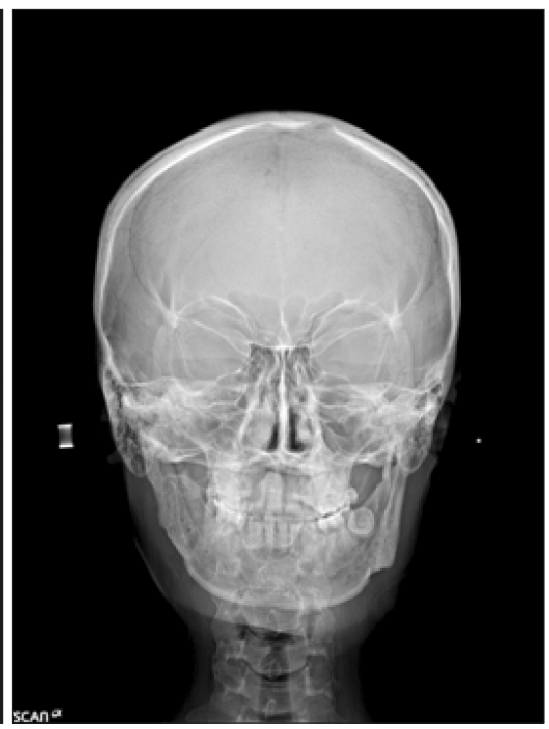

Figure 12. Post-treatment lateral cephalogram and postero-anterior cephalogram acquired after debonding. 


\begin{tabular}{|c|c|c|c|c|c|}
\hline View & $\begin{array}{c}\text { Post-surgery } \\
1 \mathrm{D}\end{array}$ & $\begin{array}{c}\text { Post-surgery } \\
1 \mathrm{M}\end{array}$ & $\begin{array}{c}\text { Post-surgery } \\
3 \mathrm{M}\end{array}$ & $\begin{array}{c}\text { Post-surgery } \\
8 \mathrm{M}\end{array}$ & $\begin{array}{c}\text { Post-surgery } \\
1 \mathrm{Y} 5 \mathrm{M}\end{array}$ \\
\hline Coronal & & & & & \\
& & & & & \\
& & & & & \\
\end{tabular}
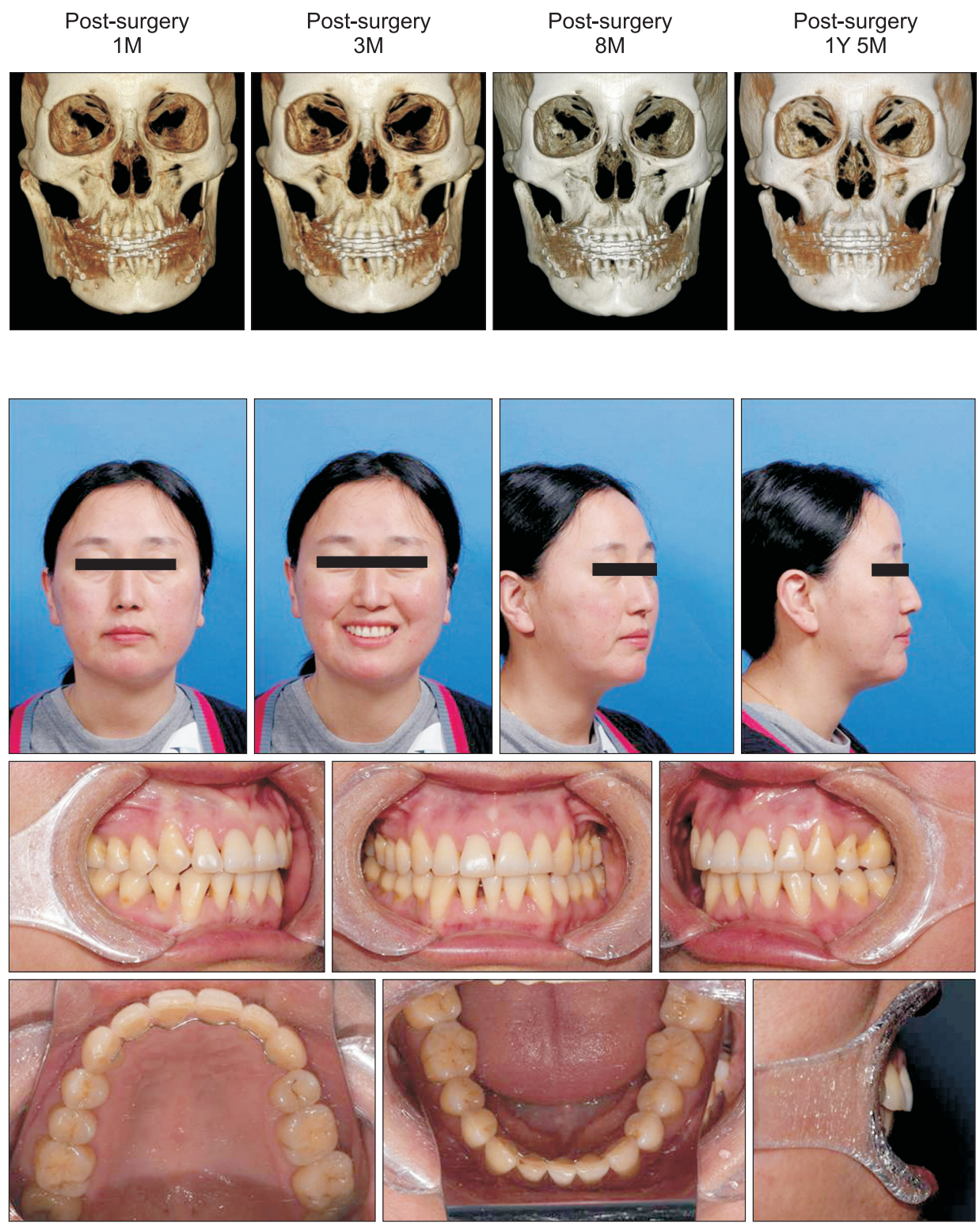

Figure 13. Serial cone-beam computed tomography images depicting the treated condyle after surgery.

1D, 1 day; $1 \mathrm{M}, 1$ month; $3 \mathrm{M}$, 3 months; $8 \mathrm{M}, 8$ months; $1 \mathrm{Y}$ $5 \mathrm{M}, 1$ year and 5 months.

Figure 14. Frontal view serial three dimensional cone-beam computed tomography images after the procedure.

$1 \mathrm{M}, 1$ month; $3 \mathrm{M}, 3$ months; $8 \mathrm{M}, 8$ months; $1 \mathrm{Y} 5 \mathrm{M}, 1$ year and 5 months.

Figure 15. Facial and intraoral photographs during the retention period (2 years). 
surgery. In CBCT, condyle and glenoid fossa remodeling were evident 3 months after the operation, moreover a round and smooth shape condyle with bone corticalization was present at 1 year and 5 months (Figure 13). This was consistent with Olate et al. ${ }^{13}$ who reported that 1 year after partial condylectomy the condyle had a round and clear cortical bone, and they also achieved a stable result. In frontal view CBCT, the affected ramus posterior region and the gonial region exhibited resorption, but the right condyle which was displaced a little laterally 1 month after the operation was slowly repositioned medially at 3 months and at 8 months after the operation (Figure 14). Appropriate occlusion and aesthetics were well maintained 2 years after the surgery (Figure 15).

\section{DISCUSSION}

Osteochondroma is often accompanied by facial asymmetry and causes functional and aesthetic problems. The etiology of osteochondroma is not yet well understood. It has been hypothesized that it is caused by osteoperiosteal deficiency induced by cartilage precursor cells, ${ }^{3}$ and that it is caused by metaplastic changes in the periosteum and osseocartilaginous layer. ${ }^{14}$

Chen et al. ${ }^{15}$ classified osteochondromas of the mandibular condyle into two types based on morphology in computed tomography imaging. Type 1 proliferates in normal condyle exophytically with directionality, and type 2 proliferates in a globular shape surrounding the normal condyle. Type 1 involves less than two-thirds of the condyle and exhibits a clear boundary; therefore, it is possible to estimate the size of the normal mandibular condyle, and a groove is created between the normal mandibular condyle and the tumor. Type 2 involves more than two-thirds of the condyle, and it is characterized mainly by skeletal deformation of the maxilla. Therefore, localized lesion resection is recommended for type 1 , and subtotal condylectomy/total condylectomy using costochondral graft reconstruction is recommended for type 2.

In CBCT in the present case, a tumor larger than 30 $\mathrm{mm}$ in diameter was observed in the antero-medial side of the condyle invading the inferior of infratemporal fossa, but it involved less than two-thirds of the condyle and exhibited a clear boundary. Thus, it was diagnosed as a gigantic form of type 1 osteochondroma developing from the antero-medial side. In cases of antero-medial gigantic osteochondroma, zygomatic arch causes a poor field of vision so determining the best surgical approach requires due consideration. We excluded an external approach, which would have entailed additional zygoma resection and scarring of the facial area. Via an intraoral approach however, the skeletal discrepancy was solved in conjunction with the removal of the osteochondroma. Good functional and aesthetic results had previously been reported using condyle contour remodeling surgery and sagittal split ramus osteotomy performed via the same oral approach. ${ }^{16}$

After removal of the osteochondroma of the mandibular condyle in the present case, remodeling and absorption of the condyle proceeded. If condyle is absorbed, the mandible is shifted backward. An open bite tends to occur, accompanied by an increase in anterior overjet and a decrease in overbite. In addition, with the mandibular clockwise rotation both the occlusal plane and the mandibular plane increase, and thereby anterior facial height increases and the facial ratio decreases.

In lateral cephalography in the present case, by 6 months postoperatively resorption of the condyle had resulted in increases in mandibular clockwise rotation and overjet, and a reduction in overbite. Intrusion of the maxillary right molars continued thereafter, with the use of TADs to compensate for clockwise rotation. After 9 months, the amount of condylar absorption gradually reduced. After 1 year and 5 months, there was almost no change in cephalograms.

\section{CONCLUSION}

Accurate diagnosis and careful treatment planning are important in cases of malocclusion associated with osteochondroma. In the current case, mandibular setback surgery using BSSRO was performed, and a tumor in the mandibular condyle was removed simultaneously via an intraoral approach. After the removal of an osteochondroma, it is difficult to accurately predict the changes in shape that will subsequently occur via condyle remodeling, and the amount of resorption that will ensue. There is also little research pertaining to long-term stability in this context available to date. Notably however, it is necessary to actively manage the ongoing clinical symptoms via an approach that is based on a good understanding of the associated occlusal changes in condyle resorption. In the present case, it was possible to achieve adequate functional and aesthetic improvements via postoperative orthodontic treatment. Nevertheless, it is necessary to monitor remodeling of the condyle and associated changes in occlusion for a long time postoperatively.

\section{CONFLICTS OF INTEREST}

No potential conflict of interest relevant to this article was reported.

\section{REFERENCES}

1. Meng Q, Chen S, Long X, Cheng Y, Deng M, Cai 
$\mathrm{H}$. The clinical and radiographic characteristics of condylar osteochondroma. Oral Surg Oral Med Oral Pathol Oral Radiol 2012;114:e66-74.

2. Cimino R, Steenks MH, Michelotti A, Farella M, PierFrancesco N. Mandibular condyle osteochondroma. Review of the literature and report of a misdiagnosed case. J Orofac Pain 2003;17:254-61.

3. Ribas Mde O, Martins WD, de Sousa MH, Zanferrari FL, Lanzoni T. Osteochondroma of the mandibular condyle: literature review and report of a case. J Contemp Dent Pract 2007;8:52-9.

4. Avinash KR, Rajagopal KV, Ramakrishnaiah RH, Carnelio S, Mahmood NS. Computed tomographic features of mandibular osteochondroma. Dentomaxillofac Radiol 2007;36:434-6.

5. Henry CH, Granite EL, Rafetto LK. Osteochondroma of the mandibular condyle: report of a case and review of the literature. J Oral Maxillofac Surg 1992;50:1102-8.

6. Karras SC, Wolford LM, Cottrell DA. Concurrent osteochondroma of the mandibular condyle and ipsilateral cranial base resulting in temperomandibular joint ankylosis: report of a case and review of the literature. J Oral Maxillofac Surg 1996;54:640-6.

7. Vezeau PJ, Fridrich KL, Vincent SD. Osteochondroma of the mandibular condyle: literature review and report of two atypical cases. J Oral Maxillofac Surg 1995;53:954-63.

8. Forssell H, Happonen RP, Forssell K, Virolainen E. Osteochondroma of the mandibular condyle. Report of a case and review of the literature. Br J Oral Maxillofac Surg 1985;23:183-9.

9. Gaines RE Jr, Lee MB, Crocker DJ. Osteochon- droma of the mandibular condyle: case report and review of the literature. J Oral Maxillofac Surg 1992;50:899-903.

10. Tanaka E, Shiota C, Sato M, Fujihara S, Kondoh T, Kuroda S. Unilateral mandibular condylar osteochondroma treated with ipsilateral condylectomy and contralateral ramus osteotomy. Am J Orthod Dentofacial Orthop 2016;149:740-50.

11. lizuka T, Schroth G, Laeng RH, Lädrach K. Osteochondroma of the mandibular condyle: report of a case. J Oral Maxillofac Surg 1996;54:495-501.

12. Miyamoto H, Shigematsu H, Suzuki S, Sakashita H. Regeneration of mandibular condyle following unilateral condylectomy in canines. J Craniomaxillofac Surg 2004;32:296-302.

13. Olate S, Cantín M, Palmieri C, Alister JP, Muñoz M, de Moraes M. Mandibular condyle repair after partial condylectomy in patients with active condylar hyperplasia. Int J Morphol 2015;33:759-63.

14. Koole R, Steenks MH, Witkamp TD, Slootweg PJ, Shaefer J. Osteochondroma of the mandibular condyle. A case report. Int J Oral Maxillofac Surg 1996; 25:203-5.

15. Chen MJ, Yang C, Qiu YT, Zhou Q, Huang D, Shi HM. Osteochondroma of the mandibular condyle: a classification system based on computed tomographic appearances. J Craniofac Surg 2014;25: 1703-6.

16. Yang JY, Leem DH. Surgical treatment of osteochondroma on the mandibular condyle through intraoral approach: case report. J Korean Assoc Maxillofac Plast Reconstr Surg 2012;34:349-56. 